\title{
Meta-analysis and systematic review of liver transplantation as an ultimate treatment option for secondary sclerosing cholangitis
}

\author{
Eyad Gadour ${ }^{1}$, Zeinab Hassan ${ }^{2}$ \\ ${ }^{1}$ University Hospitals of Morecambe Bay NHS Foundation Trust, Lancaster, United Kingdom \\ ${ }^{2}$ Faculty of Medicine, The National Ribat University, Khartoum, Sudan
}

Gastroenterology Rev 2022; 17 (1): 1-8

DOI: https://doi.org/10.5114/pg.2021.110483

Key words: secondary sclerosing cholangitis, liver transplantation.

Address for correspondence: Eyad Gadour MRCP(UK), ESEGH, FRCP (Glasg), University Hospitals of Morecambe Bay NHS Foundation Trust, Glasgow, United Kingdom, e-mail: eyadgadour@doctors.org.uk

\begin{abstract}
Introduction: Secondary sclerosing cholangitis (SSC) is a chronic cholestatic biliary disease, characterized by inflammation, obliterative fibrosis of the bile ducts, stricture formation, and progressive destruction of the biliary tree, which leads to biliary cirrhosis. In recent years, the development of secondary sclerosing cholangitis in critically ill patients (SSC-CIP) has increasingly been perceived as a separate disease entity.

Aim: To perform a systematic review and meta-analysis of secondary sclerosing cholangitis and ischaemic cholangiopathy in post organ transplant patients and intensive care unit (ICU).

Material and methods: A comprehensive search strategy using the PubMed, Biosis, and EMBASE databases was designed to retrieve relevant clinical data from the published literature up to 2020. Demographic characteristics, laboratory, transplantation, mortality rate, and follow-up data undergoing liver transplantation were extracted from the inclusion studies. We used DerSimonian-Laird random-effects meta-analysis. Analysis was carried out using R statistical software version 4.02.

Results: A total of 862 patients with SSC-CIP were extracted from 16 studies. Eighteen studies were searched for the meta-analysis, out of which 16 studies were eligible for the meta-analysis and 2 were excluded. A proportion meta-analysis was performed on liver transplant patients with SSC-CIP and on mortality rate. Significant results were found (Prop $=0.30,95 \% \mathrm{Cl}$ : $0.12-0.49, p<0.01)$, with high heterogeneity among the studies $\left(I^{2}=98 \%, p<0.01\right)$ and (Prop $=0.45 ; 95 \% \mathrm{Cl}: 0.35-0.56$ with $R^{2}=80, p<0.01$ ), respectively. No indication of publication bias, as confirmed by the funnel plot and the risk of bias in the included studies, shows that the study reporting is adequate to judge that no major or minor sources of bias are likely to influence results.

Conclusions: The systematic review and meta-analysis show that liver transplantation is a valid option for patients with SSC-CIP, with excellent long-term outcome and improvement of quality of life.
\end{abstract}

\section{Introduction}

Secondary sclerosing cholangitis (SSC) is a chronic cholestatic biliary disease, characterized by inflammation, obliterative fibrosis of the bile ducts, stricture formation, and progressive destruction of the biliary tree, which leads to biliary cirrhosis [1]. The most frequently described causes of SSC are longstanding biliary obstruction, surgical trauma to the bile duct, and ischaemic injury to the biliary tree in liver allografts [1]. SSC in critically ill patients (SSC-CIP) requiring aggressive intensive care treatment is a largely unrecognized new form of SSC, associated with rapid pro- gression to liver cirrhosis. The mechanisms leading to the cholangiopathy in critically ill patients are widely unknown; however, it is probably mediated through ischaemic injury to the bile ducts [1]. Unlike hepatic parenchyma, which depends on a dual blood supply from the hepatic artery and portal vein, the biliary system depends only on its arterial supply for viability [2]. Arterial hypotension of the peribiliary vascular plexus causes ischaemic injury of the bile duct epithelium [1]. The available clinical data indicate that ischaemic injury to the intrahepatic biliary tree followed by bacterial colonization leading to destructive biliary changes may be one of the earliest events in the development of 
this severe form of SSC-CIP [3]. These patients usually have no prior history of biliary disease or liver disease and no evidence of a pathological process or injury that has obviously caused obstruction of the bile duct [4-10]. The clinical and radiological manifestations reflect cholestasis [4-10]. Cholangiographic findings resemble those of primary sclerosing cholangitis. Therapeutic options for most forms of SSC-CIP are limited, and patients who do not undergo transplantation have rapid disease progression and poor outcome [1, 11]. Current understanding and management of SSC-CIP is limited. Only 8 patients with SSC-CIP following major burn injury were described previously $[5,8,10,11]$. SSC-CIP is a relatively new previously unrecognized entity, which may lead to severe biliary disease with rapid progression to cirrhosis. Medical and technical progress in critical care medicine has increased survival rates of patients with life-threatening injuries. However, as critically ill patients more often survive, new intensive care unit (ICU)-acquired diseases have emerged. Recent years have seen increasing reports of a new entity of ICU-associated diseases. The development of a SSC-CIP was first observed in patients suffering burns or polytrauma, but subsequently also following other acute life-threatening events [1-4]. SSC-CIP is characterized by cholestasis and bile duct necrosis and can be diagnosed on the basis of typical cholangiographic findings. It appears to be irreversible and progresses rapidly to biliary cirrhosis and liver failure. Without exception, the medical history of all reported patients with SSC-CIP revealed previous intensive care treatment [12-15]. There is no doubt that the triggers of SSC-CIP are to be found in the time window between the life-threatening initial event and the occurrence of cholestasis. Whether the disease is caused by the initial injury itself or by the intensive care treatment is not yet clear. Therapeutic options for SSC-CIP are limited, the mortality rate during ICU stay is high, and many surviving patients must undergo liver transplantation. If ICU-associated causes could be identified, appropriate avoidance strategies might help prevent the development of SSC-CIP. Currently available reports speculate on a range of possible causes of SSC-CIP, such as sepsis, high-dose catecholamines, and ischaemia $[13,14]$.

\section{Aim}

We present for the first time a systematic review and meta-analysis of secondary sclerosing cholangitis and ischaemic cholangiopathy in post organ transplant patients and ICU. The methodology, data extraction, meta-analysis, as well as the results and outcome of this study are discussed.

\section{Material and methods}

Records of (862) patients who received or were listed for liver transplantation (LT) extracted from 16 studies, at the chosen hospital, were carefully reviewed. A total of 126 patients with a confirmed diagnosis of SSC-CIP were identified. The diagnosis of SSC-CIP was based on typical findings on endoscopic retrograde cholangiography (ERC), such as the presence of casts or destruction of intrahepatic bile duct branches. Other causes of cholestasis were excluded by comprehensive differential diagnostic examinations. Additionally, liver histology was available in 126 patients. As tertiary care centres, the Departments of Transplantation Surgery and Gastroenterology receive patients for liver transplantation as well as for endoscopic or percutaneous biliary interventions from various other hospitals. Therefore, most of our patients underwent initial intensive care treatment at outside hospitals.

\section{Search strategy and selection criteria}

A comprehensive search strategy using the PubMed, Biosis, and EMBASE databases was designed to retrieve relevant clinical data from the published literature up to 2020. The following search terms were pre-defined: "sclerosing”, "ischaemic”, "SSC", "CIP”, "Liver”, "transplant”, "cholangitis* or colangitis* or cholang”, “ill”, "critical", "diagnosis", "patients or patient", and "aetiology database".

Two independent authors selected studies and extracted data. Disagreements were resolved by discussion between the 2 reviewers. This analysis used broad inclusion criteria to comprehensively capture all relevant data. Inclusion and exclusion criteria are specified.

\section{Data extraction}

The following information were extracted from each study: (1) study identity; (2) number of patients studied for SSC-CIP; (3) number of patients for liver transplanting; (4) number of mortalities; (5) length of follow-up (months); (6) methods of assay; (7) donation after brain death; (8) ischaemic cholangitis. Clinical SSC-CIP and liver transplanting were defined according to the criteria adopted by each study.

\section{Study inclusion}

Eighteen studies were searched for the meta-analysis, out of which 16 were eligible for the meta-analysis [4-6, 10, 12, 14-24].

\section{Study exclusion}

Two studies were excluded from the meta-analysis, one study reported the aetiology and risk factor of isch- 
emic cholangitis (IC) after liver transplantation, which is beyond the scope of study, and the other study contained no required information needed for the analysis.

\section{Statistical analysis}

Secondary and primary sclerosing cholangitis (PSC) and ischaemic in post organ transplant patients has been classically attributed to a chronic disease, and many studies investigated the prevalence only among PSC patients. To clarify the association of ICU development to transplant responsiveness, this analysis considered 3 principal categories of patients: all patients, IC patients, and donation after circulatory death (DCD) and donation after brain death (DBD) patients. We used DerSimonian-Laird random-effects meta-analysis to summarize the effect of liver transplanting for both DCD and DBD patients, the mortality rates among patient (with 95\% confidence intervals Cls). For all meta-analyses, prevalence estimates were transformed to logits to improve their statistical properties, and later reverse transformed. We assessed the heterogeneity of prevalence estimates between studies using the $I^{2}$ statistics. The overall effects across all studies were estimated with $95 \%$ confidence intervals. The publication bias was access using funnel plots, and the risk of bias graph and summary were assessed using RevMan 5.4. All analyses were carried out using $R$ statistical software version 4.02 .

\section{Results}

SSC-CIP is a progressive and destructive cholangiopathy with a grim prognosis. Data on the incidence of SSC-CIP were extracted for patients with liver trans- plantation; mortality rate and some factors that lead to SSC, such as ischaemia, were extracted from 16 studies and analysed.

The global distribution of the reported cases was of high importance. Although the cases of SC-CIP were reported by researchers from different regions worldwide, the greatest portion (97.1\%) was reported by centres located in Germany; others were in the Netherlands and Israel (10 articles, including 87 cases; Table I).

\section{Meta-analysis}

Thirteen studies reported SSC-CIP rates for liver transplantation. A significant result was found (Prop $=0.30$, 95\% Cl: 0.12-0.49, $p<0.01$ ), which implies successful treatment. Overall heterogeneity was significant $\left(I^{2}=\right.$ $98 \%, p<0.01)$, which implies that the heterogeneity among the studies were high, but this decreased when examining only hospitalized patients not in ICUs and tended to zero in the sensitivity analyses for the general populations (Figure 1). As a countercheck to our observations on mortality, we performed a meta-analysis on those papers describing mortality rates for SSC-CIP (Figure 2). Twelve studies were included, with quality ranging from satisfactory to very good: the pooled effect size displaying a significant result was also found (Prop $=0.45$; $95 \% \mathrm{Cl}: 0.35-0.56)$. Heterogeneity of the included studies was modest $\left(R^{2}=80\right)$ but statistically significant $(p<0.01)$.

In a study in which there is no publication bias, larger studies (i.e. with lower standard error) tend to cluster closely to the point estimate. As studies become less precise, such as in smaller trials (i.e. with a higher standard error), the results of the studies can be expected to be more variable and are scattered to both sides of

Table I. Summary of SC-CIP cases reported in included studies

\begin{tabular}{|c|c|c|c|c|}
\hline Year & Author & Country & No. of patients (male/female) & Age \\
\hline 2001 & Scheppach et al. [15] & Germany & $3(1 / 2)$ & 32.02 \\
\hline 2012 & Siebers et al. [18] & Netherland & $1(0 / 1)$ & 48 \\
\hline 2003 & Engler et al. [4] & Germany & $9(5 / 4)$ & 56.2 \\
\hline 2005 & Benninger et al. [5] & Germany & $7(5 / 1)$ & 48.4 \\
\hline 2006 & Jaeger et al. [14] & Germany & $10(5 / 5)$ & 55.02 \\
\hline 2007 & Gelbmann et al. [12] & Germany & $26(20 / 6)$ & 46.66 \\
\hline 2008 & Kulaksiz et al. [6] & Germany & $29(21 / 8)$ & 52.2 \\
\hline 2008 & Esposito et al. [10] & Germany & $10(9 / 1)$ & 49.7 \\
\hline 2009 & Schnitzbauer et al. [25] & Germany & $1(1 / 0)$ & 30 \\
\hline 2015 & Ben-Ari et al. [19] & Israel & $1(1 / 0)$ & 48 \\
\hline 2015 & Leonhardt et al. [20] & Germany & $1(1 / 0)$ & 22 \\
\hline 2012 & Voigtlander et al. [9] & Germany & $1(1 / 0)$ & 68 \\
\hline Total & - & - & $99(67 / 28)$ & 48.7 \\
\hline
\end{tabular}




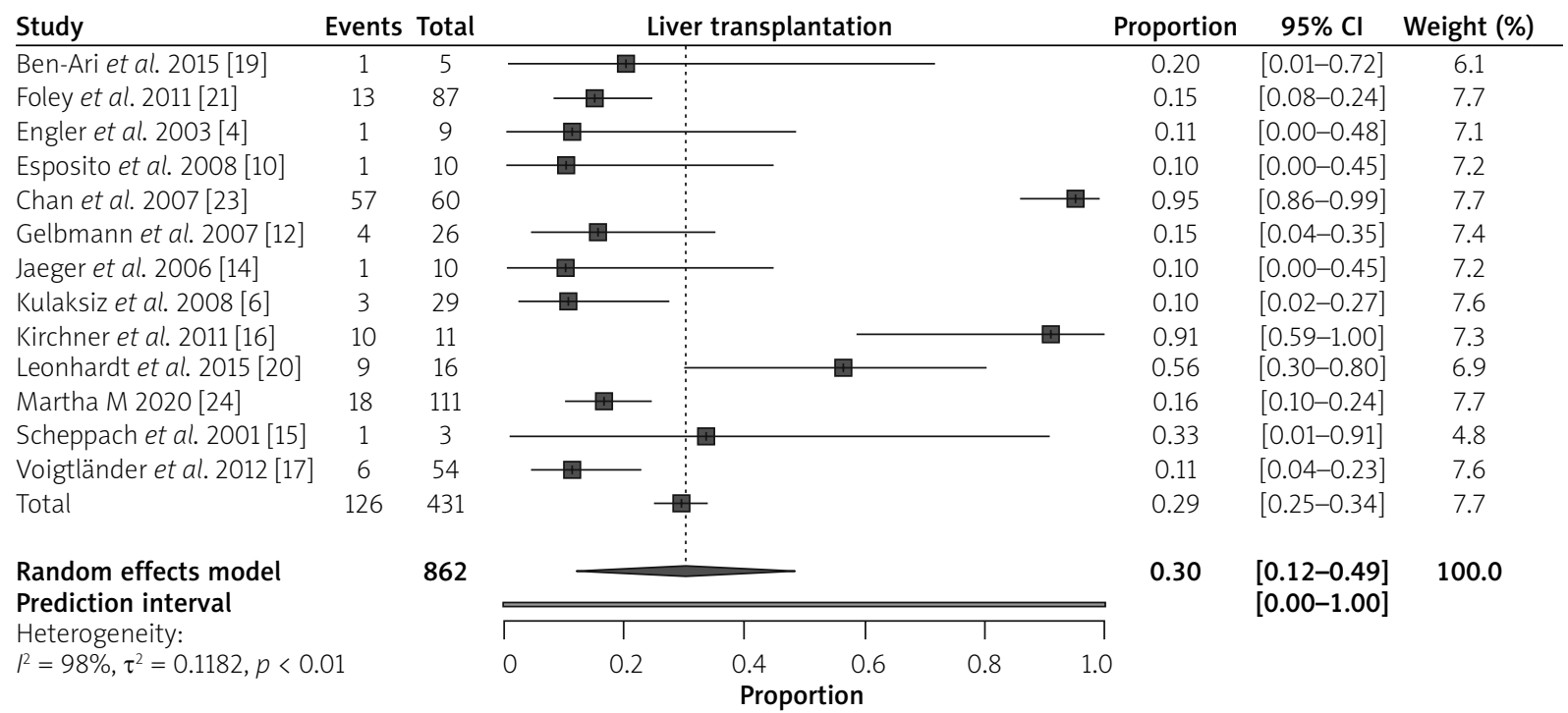

Figure 1. Forest plot of SSC-CIP (sclerosing cholangitis in critical ill patients with liver transplant)

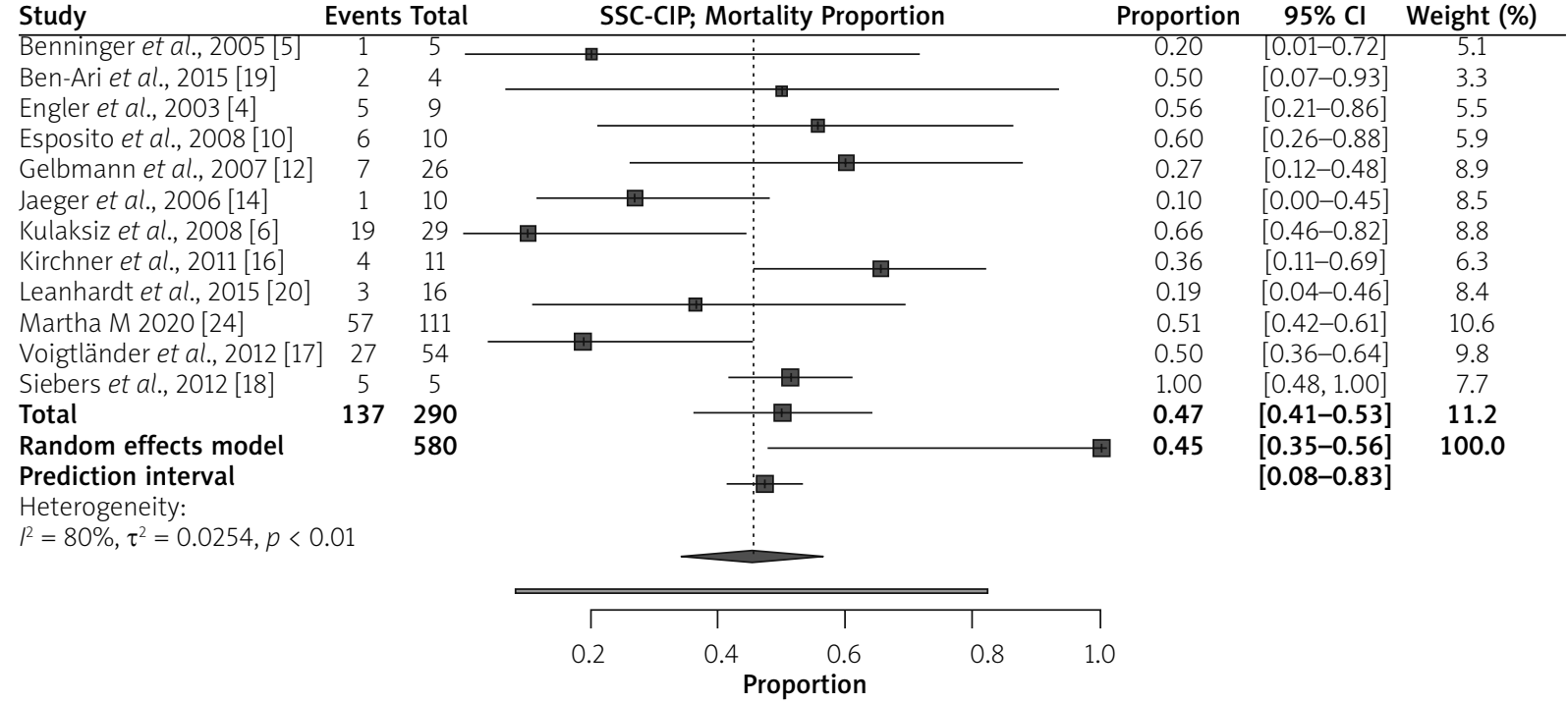

Figure 2. Forest plot of SSC-CIP mortality rate among patients who underwent LT

the more precise larger studies. Two funnel plots were plotted for studies that reported liver transplantation (LT) and mortality with SSC-CIP (Figures 3 and 4); the points were symmetrical, which shows no evidence of publication bias.

Figures 2 and 5 show that the smaller, less precise studies are, indeed, scattered to both sides of the point estimate of effect, and that these seem to be symmetrical, as an inverted funnel-plot, showing no evidence of publication bias.

\section{Risk of bias}

Figure 5 shows the risk of bias summaries and graph; the white colour indicates an unclear risk, the red indicates high risk, while the green colour represents low risk. In the first figures, a study categorized as low risk of bias implies confidence on the part of the reviewer that the results represent the true treatment effects (study results are considered valid). The study reporting is adequate to judge that no major or minor sources of bias are likely to influence the results. A study categorized as high risk of bias implies low confidence that results represent true treatment effect. The study has significant flaws that imply biases of various types that may invalidate its results; these may arise from serious errors in conduct, analysis, or reporting, large amounts of missing information, or discrepancies in reporting. A study categorized as "unclear" risk of 


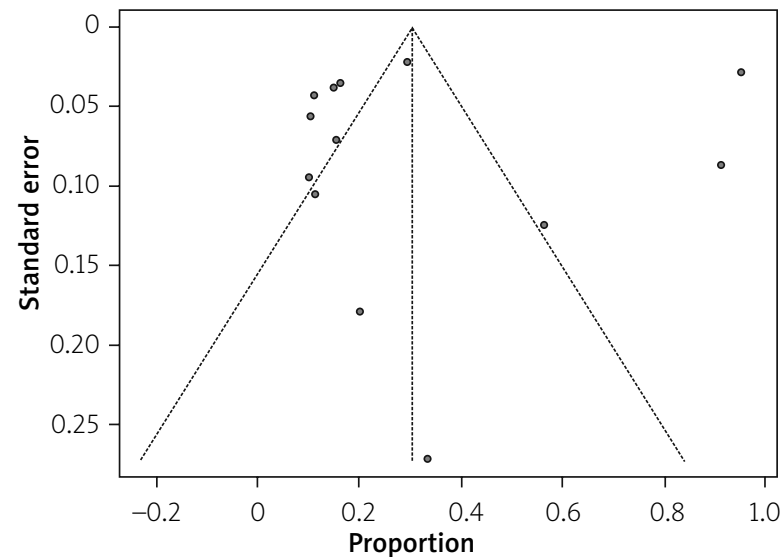

Figure 3. The funnel plot of SSC-CIP (sclerosing cholangitis in critical ill patient) with liver transplanting showing publication bias

bias is missing information, making it difficult to assess limitations and potential problems. We can say that all included studies represent the true treatment effect (liver transplant for SSC-CIP).

\section{Discussion}

SSC is a rare, rapidly advancing group of chronic cholestatic diseases involving biliary tree inflammation, both intra and/or extrahepatic, and progressing to form stricture, which leads to biliary cirrhosis. SSC-CIP is a new and more acknowledged term due to prolonged ICU stay and treatment due to unknown cause of liver injury. Liver transplantation is probably a curative therapeutic option for patients with liver cirrhosis induced by SC-CIP. Few data are available on the outcome of SC-CIP patients after liver transplantation. The SSC-CIP is a severe condition and has the potential to develop liver cirrhosis with limited medicinal and procedural options with poor prognosis and low patient survival $[16,17]$. During the initial stages of the disease, the clinical symptoms and biochemical profile are not specific and are easily missed. SSC-CIP is difficult to diagnose in the early phase of disease development; clinical symptoms, gut permeability markers, and blood testing were also non-specific [16, 17]. Biochemical profile and other diagnostic tests are helpful when the disease progresses, and they are crucial in decision planning of liver transplantation [16]. Biliary cast formation is the early pathognomonic indication of SSC-CIP and crucial for identification of disease progression and for liver transplantation decision [16].

Liver cirrhosis is interpreted with $>14.3 \mathrm{kPa}$ with the cut-off of $8.8 \mathrm{kPa}$ liver fibrosis by transient elastography or Fibroscan [17]. There are some potential associated factors such as cardiogenic shock, polytrau-

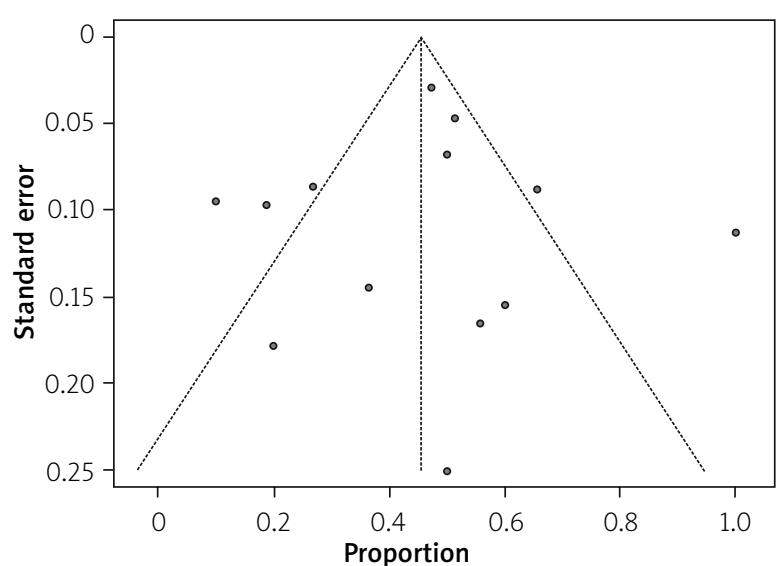

Figure 4. The funnel plot of SSC-CIP. Mortality showing publication bias

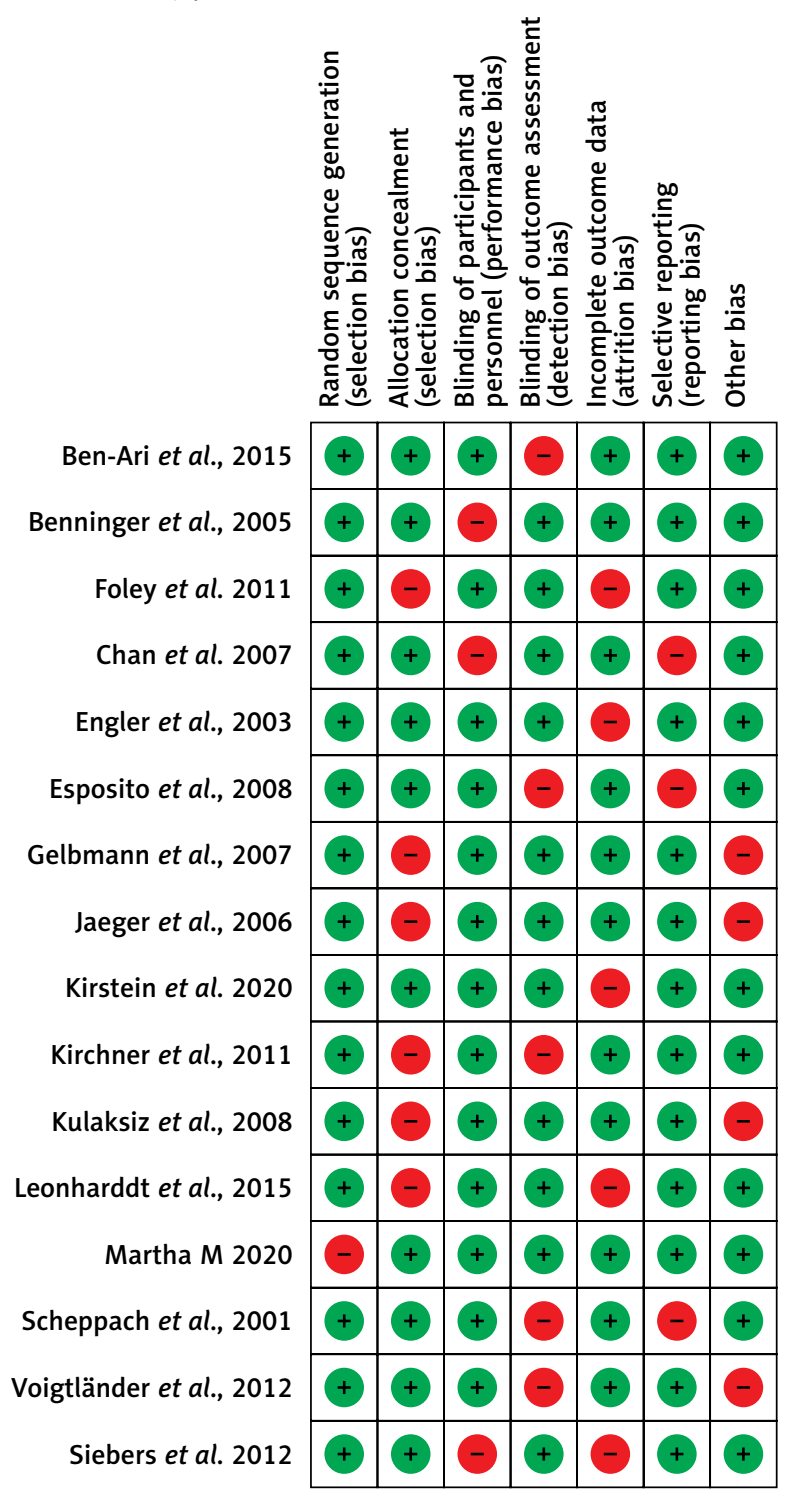

Figure 5. Risk of bias summary for all included studies 
ma, ischaemia, surgeries, burns, toxic and infectious immunological processes, chronic biliary obstruction, mechanical ventilation, hypovolaemic shock, and associated comorbidities like obesity [17]. Some morphologic characteristics associated with ITU-SSC have in part been described in previous studies and correlate well with our findings. In 2 of the 3 cases reported by Scheppach et al., a liver biopsy was performed 8 and 11 months after initial symptoms, respectively, and in cases, ductular proliferation and bridging fibrosis was present. The third patient underwent liver transplantation, and the examination of the explanted organ showed PSC-like changes, including ulceration of the epithelium of large intrahepatic bile ducts, which were filled with bile plugs [25]

Ultrasound reveals the presence of inhomogeneous hepatic parenchyma and its severity if there is any indication of liver cirrhosis, like the presence of nodule, deformity of the liver, and segmental dilation of the bile duct. Magnetic resonance imaging (MRI) reveals liver atrophy and intrahepatic segmental dilation. Endoscopic retrograde cholangiopancreatography (ERCP) is used to evaluate any change that occurs in the intrahepatic bile duct [5]. The only recommended treatment yet is the liver transplantation [16].

We performed for the first time a systematic meta-analysis of secondary sclerosing cholangitis and ischaemic cholangiopathy in post organ transplant patients and ICU from 16 studies, of which some reported liver transplants in both DCD and DBD; some studies also reported the mortality of patients with SSC-CIP.

The mean duration of SSC-CIP development after ITU stay is 30 to 40 days, but rare cases of SSCCIP progression were also reported at 9 days only [17]. Treatment therapeutics in the initial disease phase are cyclosporine, penicillamine, cladribine, budesonide, colchicine, etanercept, methotrexate, infliximab, pentoxifylline, mycophenolate mofetil, nicotine, pirfenidone, silymarin, and tacrolimus. In the case of bacterial involvement, antibiotic treatment and biliary casts by endoscopy can occasionally be helpful, depending upon the patient's status [17]. A significant result was found in the meta-analysis carried out on patients with LT studies $[6,10,12-14,17,19,21,24]$, showing a significant result with $p<0.01$, which implies successful treatment, as reported in the studies, regarding the patients' response to treatment in both DCD and DBD liver transplant. Among the studies, there was a high percentage heterogeneity $\left({ }^{2}=98 \%\right)$ with $(\tan 2$ value $=$ $0.1182)$; however, some studies $[15,16,23]$ were slightly diverted from the line of no difference to the right, which implies insignificant results (prop.: $0.95,95 \% \mathrm{Cl}$ : 0.86-0.99, prop: $0.91,95 \% \mathrm{Cl}: 0.59-1.00$, prop: 0.56 ,
95\% Cl: 0.30-0.80, prop: 0.33, 95\% Cl: 0.01-0.91), but the overall random effect model was highly significant (prop: 0.30, 95\% Cl: 0.12-0.49) (Figure 1).

The second forest plots show a significant result in the mortality rate: it shows high mortality among patients with SSC-CIP with ( $p<0.01$, prop: $0.45,95 \% \mathrm{Cl}$ : $0.35-0.56)$, the heterogeneity among the studies that reported the number of mortalities is low when compared to the first forest plot $\left(I^{2}=80 \%, \tan 2=0.0254\right)$ (Figure 2).

The disease progression towards liver damage leaves liver transplantation as the only choice $[18,19]$. Ninety-eight percent of SSC-CIP cases were positive for bacterial or fungal infection, as reported in the collected bile samples. Enterococcus faecium or Enterococcus faecalis accounted for $71 \%$ of infections while methicillin-resistant Staphylococcus aureus (MRSA), Pseudomonas aeruginosa, Stenotrophomonas maltophilia, Lactobacillus, and Blautia are the remaining causative agents of bacterial presence. Among fungal isolates, Candida is responsible for most of the infections [16, 17]. These bacterial and fungal isolates are mostly multi-drug resistant (MDR) and are difficult to treat with antibiotics [16]. The reported gut microbiome modification of SSC-CIP patients was also evaluated, and the reported bacterial strains are Enterococcus, Atopobium, Bifidobacterium, Coprococcus, Lachnospira, Lactobacillus, Lactococcus, Rothia, Ruminiclostridium, Ruminococcaceae, Ruminococcaceae, Sellimonas, Solobacterium, Sutterella, Streptococcus thermophilus, Streptococcus parasanguinis, Rothiadentocariosa, Sellimonas intestinalis, and Anaerostipeshadrus [17]. Diagnostic workup is also important to plan for liver transplantation, including blood, biopsy, and radiological testing. Blood testing includes liver enzymes elevation, renal function tests, platelet and total leucocyte count, and estimated sedimentation rate (ESR), and gut permeability markers include calprotectin, zonulin, diamine oxidase (DAO), lipopolysaccharide-binding protein (LPB), and soluble CD14 (sCD14) levels.

Histological findings of liver biopsy, radiological imaging, ultrasound testing, Doppler ultrasound, computed tomography (CT), MRI, and ERCP [5, 22], and elastography for evaluation of liver stiffness. ERCP is considered in order to conclude the diagnosis in most cases, and it is the gold standard diagnostic test for SSC-CIP $[16,17]$. Ultrasound and liver biopsies are also very helpful with 30 and $36 \%$ accuracy, respectively [16]. Magnetic resonance cholangiopancreatography (MRCP) has greater accuracy and efficiency, and in particular noninvasive methodology, because critically ill patients possibly have greater risk for invasive testing [16]. 
An initial 6-month period is very important to monitor for the development of imaging features of liver cirrhosis, because $88 \%$ of SSC-CIP patients present with positive testing on ERCP with liver cirrhosis indication [16]. However, associated comorbidities are another consideration before planning for liver transplantation. Patients having any comorbidities like cardiac arrhythmias, hypertension, and obesity in addition to SSC-CIP are at greater risk of liver transplantation $[17,20]$.

Two funnel plots were plotted for studies that reported LT and mortality with SSC-CIP (Figures 3 and 4). The points were symmetrical, which shows no evidence of publication bias. The risk of bias (ROB) for included studies were access using selection bias, performance bias, attrition bias, reporting bias, and other bias as unclear risk were selected, which implies that the studies are adequate to judge that no major or minor source of bias is likely to influence the results.

The decision to plan liver transplantation is crucial. This step should be initiated before disease advancement towards the complete liver cirrhosis, this is very important to prolong long-term survival outcome [19]. Liver transplantation is probably a curative therapeutic option for patients with liver cirrhosis induced by SCCIP. Few data are available on the outcome of SC-CIP patients after liver transplantation. There are certain prognostic indicators to make a decision for transplantation. One of the top listed indicators is recurrent cholangitis of bacterial origin despite intensive care, medications and associated improvement, and endoscopic treatment, severe obstruction of an extrahepatic biliary tract that urges surgical repair, unsuppressed vaginal blood loss, and uncontrolled pruritus [19]. Kirstein et al. described 11 patients who had undergone liver transplantation due to SC-CIP [24]. The 1-, 3-, and 5-year survival rates of patients with SC-CIP were $64 \%$ each. Four of the 11 patients died within the first 8 months after liver transplantation. One female patient died due to sepsis within 26 days after surgery. Ten of 11 patients were discharged from the hospital after liver transplantation; however, 2 patients died within 4 and 8 months due to sepsis, and 1 patient died 1.5 months after liver transplantation due to shock after a rupture of a hepatic artery aneurysm. In the non-traumatic SC-CIP group, sepsis was the main cause of death [22]. Patients who survived the first 8 months after liver transplantation had a good long-term survival prognosis. Studies also reported some novel methods for better diagnostics and therapeutics of SSC-CIP patients. MicroRNAs testing in serum and bile of SSC-CIP patients is a unique approach towards better outcomes, yet more evaluation is required [23]. Monitoring of gut microbiome changes may be a promising indicator for SSC-CIP development
[17]. The increase in the detection of SSC-CIP may be a result of the growing awareness of the disease and the improved therapeutic strategies in the ITU that may lead to better survival of patients with novel complications of ITU treatment (e.g. development of sclerosing cholangitis [9].

However, liver transplantation is the only treatment currently available to lower the mortality rate among SSC-CIP patients and improve the survival rate [16, 18]. Liver transplantation prolongs the survival of SSC-CIP patients with the possible recurrence of $5-35 \%$ in the transplanted grafts [22].

\section{Conclusions}

The successful treatment of SSC-CIP with liver transplantation has contributed to a decrease in the mortality rate and prolonged survival rate, although a special review of the indication for liver transplant should be discussed and picked up in the early stage of the disease. All included studies have no publication bias, as indicated by the funnel plot.

\section{Conflict of interest}

The authors declare no conflict of interest.

\section{Reference}

1. Ruemmele P, Hofstaedter F, Gelbmann CM. Secondary sclerosing cholangitis. Nat Rev Gastroenterol Hepatol 2009; 6: 287-295.

2. Batts KP. Ischemic cholangitis. Mayo Clin Proc 1998; 73: 380-5.

3. Suyapoh W, Tirnitz-Parker J, Tangkawattana S, et al. Biliary migration, colonization, and pathogenesis of O. viverrini co-infected with CagA+ Helicobacter pylori. Pathogens 2021; 10: 1089.

4. Engler S, Elsing C, Flechtenmacher C, et al. Progressive sclerosing cholangitis after septic shock: a new variant of vanishing bile duct disorders. Gut 2003; 52: 688-93.

5. Benninger J, Grobholz R, Oeztuerk Y, et al. Sclerosing cholangitis following severe trauma: description of a remarkable disease entity with emphasis on possible pathophysiologic mechanisms. World J Gastroenterol 2005; 11: 4199-205.

6. Kulaksiz H, Heuberger D, Engler S, Stiehl A. Poor outcome in progressive sclerosing cholangitis after septic shock. Endoscopy $2008 ; 40: 214-8$.

7. Lin T, Qu K, Xu X, et al. Sclerosing cholangitis in critically ill patients: an important and easily ignored problem based on a German experience. Front Med 2014; 8: 118-26.

8. Al-Benna S, Willert J, Steinau HU, Steinstraesser L. Secondary sclerosing cholangitis, following major burn injury. Burns 2010; 36: e106-10.

9. Voigtländer T, Negm AA, Schneider AS, et al. Secondary sclerosing cholangitis in critically ill patients: model of end-stage liver disease score and renal function predict outcome. Endoscopy 2012; 44: 1055-8. 
10. Esposito I, Kubisova A, Stiehl A, et al. Secondary sclerosing cholangitis after intensive care unit treatment: clues to the histopathological differential diagnosis. Virchows Arch 2008; 453: 339-45.

11. Schmitt M, Kölbel CB, Müller MK, et al. Sclerosing cholangitis after burn injury. Z Gastroenterol 1997; 35: 929-34.

12. Gelbmann CM, Rümmele P, Wimmer $M$, et al. Ischemic-like cholangiopathy with secondary sclerosing cholangitis in critically ill patients. Am J Gastroenterol 2007; 102: 1221-9.

13. Engler S, Elsing C, Flechtenmacher $\mathrm{C}$, et al. Progressive sclerosing cholangitis after septic shock: a new variant of vanishing bile duct disorders. Gut 2003; 52: 688-93.

14. Jaeger C, Mayer G, Henrich R, et al. Secondary sclerosing cholangitis after long-term treatment in an intensive care unit clinical presentation, endoscopic findings, treatment, and follow-up. Endoscopy 2006; 38: 730-4.

15. Scheppach W, Druge G, Wittenberg G, et al. Sclerosing cholangitis and liver cirrhosis after extrabiliary infections: report on three cases. Crit Care Med 2001; 29: 438-41.

16. Kirchner G, Klingsiek E, Hartl J, et al. Natural history of sclerosing cholangitis in critically ill patients (SC-CIP): predictors and outcome. J Hepatol 2013; 58 (Suppl 1): S386-7.

17. Voigtländer T, Gupta SK, Thum S, et al. MicroRNAs in serum and bile of patients with primary sclerosing cholangitis and/or cholangiocarcinoma. PLoS One 2015; 10: e0139305.

18. Siebers C, Kinzinger J, Hinske LC, et al. Effect of intraoperative single-shot application of vancomycin in liver transplant recipients on postoperative infections with Enterococcus faecium and Enterococcus faecalis. Exp Clin Transplant 2018; 16 : 701-7.

19. Ben-Ari Y, Gaiarsa JL, Tyzio R, et al. GABA: a pioneer transmitter that excites immature neurons and generates primitive oscillations. Physiol Rev 2007; 87: 1215-84.

20. Leonhardt S, Veltzke-Schlieker W, Adler A, et al. secondary sclerosing cholangitis in critically ill patients: clinical presentation, cholangiographic features, natural history, and outcome: a series of 16 cases. Medicine 2015; 94: e2188.

21. Foley DP, Fernandez LA, Leverson G, et al. Biliary complications after liver transplantation from donation after cardiac death donors: an analysis of risk factors and long-term outcomes from a single center. Ann Surg 2011; 253: 817-25.

22. Kirstein MM, Book T, Manns MP, et al. Secondary sclerosing cholangitis in critically ill patients has a poor outcome but lower tumour incidence than primary sclerosing cholangitis. United European Gastroenterol J 2020; 8: 716-24.

23. Chan EY, Larson AM, Gernsheimer TB, et al. Recipient and donor factors influence the incidence of graft-vs.-host disease in liver transplant patients. Liver Transpl 2007; 13: 516-22.

24. Kirstein MM, Scheiner B, Marwede T, et al. Sequential systemic treatment in patients with hepatocellular carcinoma. Aliment Pharmacol Ther 2020; 52: 205-12.

25. Schnitzbauer AA, Zuelke C, Graeb C, et al. A prospective randomised, open-labeled, trial comparing sirolimus-containing versus $m$ TOR-inhibitor-free immunosuppression in patients undergoing liver transplantation for hepatocellular carcinoma. BMC Cancer 2010; 10: 190.
Received: 19.01.2021

Accepted: 28.02 .2021 